\title{
HOW TO FOSTER INNOVATION? A METHODOLOGY TO IDENTIFY FIELDS FOR FOSTERING INNOVATION CAPABILITY IN SMALL AND MEDIUM-SIZED ENTERPRISES
}

\author{
Şahin, Tarık (1); Riedel, Leonhard (2); Inkermann, David (1); Wilgeroth, Dagmar (2); Asghari, \\ Reza (2); Vietor, Thomas (1)
}

1: Technische Universität Braunschweig; 2: Ostfalia University of Applied Sciences

\begin{abstract}
Small and medium-sized enterprises are affected by changing development, production and selling paradigms in globalized industries, where innovation is a driver for sustainable competitiveness. However, innovating is highly challenging for small and medium-sized enterprises, as human resources are very limited and know-how are often highly specialized. It is often unclear which fields and factors provide the capability to innovate and which measures or methods can be applied to promote innovation based on existing competencies.

For this reason, the aim of this paper is to present a framework to support the innovation capability of SMEs by identifying promising fields for innovation and providing suitable innovation methods. A particular focus in this paper is a methodology for the description and identification of fields to foster the innovation capability in SMEs as a part of the introduced framework.
\end{abstract}

Keywords: Innovation, Small and Medium-Sized Enterprises, New product development, Design methods

Contact:

Şahin, Tarık

Technische Universität Braunschweig

Institute for Engineering Design

Germany

tarik.sahin@tu-braunschweig.de

Cite this article: Şahin, T., Riedel, L., Inkermann, D., Wilgeroth, D., Asghari, R., Vietor, T. (2019) 'How to Foster

Innovation? A Methodology to Identify Fields for Fostering Innovation Capability in Small and Medium-Sized Enterprises', in Proceedings of the 22nd International Conference on Engineering Design (ICED19), Delft, The Netherlands, 5-8 August 2019. DOI:10.1017/dsi.2019.233 


\section{INTRODUCTION}

Increasing saturation and intensifying competitive conditions in globalized markets demand shorter product life cycles and rising product varieties in the manufacturing industry. The continuous development of innovative products is becoming a decisive and challenging key factor - the capacity of competitiveness is thus becoming a question of the capability to innovate. However, many companies struggle to bring out innovation continuously and systematically. A high rate of innovations arise within subjective circumstances and often by chance consequently by high risks (Cooper, 2001). Methods are valuable means to bring out innovation respectively in order to handle related risks and exploit potentials respectively (Eversheim, 2009). In particular, small and medium-sized enterprises (SMEs) are faced with great challenges in the field of innovation due to their characteristics, such as limited resources and restricted methodological knowledge, compared to large companies with the capability to conduct innovation activities in a more systematical way (Rosenbusch et al., 2011). Potentials for innovation based on high technological knowledge within SMEs are thus unexploited and hindered by a diffused understanding of fields and methods for innovation (Hidalgo and Albors, 2008). The authors therefore highlight the need for consistent concepts to support the identification of fields and methods to foster innovation capabilities of SMEs.

The main objective of this contribution is to discuss findings about relevance and successful application of innovation methods and to introduce of a corresponding methodology to identify fields and methods to foster innovation capabilities of SMEs. In order to highlight the need and motivation for a corresponding methodology, we discuss the relevance and current practice of method application in the field of innovation focusing on SMEs in the following section. Based on the current challenges in practice we propose a framework for successful method application in manufacturing SMEs in the field of innovation. As a part of the framework a methodology and according model supporting the identification of fields to foster innovation capability in SMEs is introduced with insights about its practical application. In addition, fields to foster innovation capability of SMEs are introduced as a main result. Respective finding for further research are discussed based on the development and concluding application of the methodology in different manufacturing SMEs.

\subsection{Relevance of method application to foster innovation capabilites of SMEs}

A diffused understanding can be observed in practice as well as in literature in the field of innovation and its related methods. Several definitions of innovation from different disciplines are described by various dimensions and characteristics (Baregheh et al., 2009). The characteristics of the term are often interdepending and thus definitions are never absolute and have no clear borderlines (Cooper, 1998). A generic definition of innovation is the realization of a novel idea with successful diffusion in the market (Schumpeter, 1934). The purpose of defining innovation is not to end the discussion on what describes innovation, but to have a frame and a focus in context of its application or research. However, as innovation is crucial for long-term success in SMEs a deeper understanding of the capability to bring out innovation and its drivers within the corporate systems is required. Two main perspectives on managing innovation capability are described in literature (Bleicher, 1999; Eversheim, 2009). The first perspective is process-oriented and focuses on the sequential steps of innovation activities. The second perspective highlights a system-theoretic view and denotes that innovation capability is a result of alignment in terms of corporate structures, innovation activities and innovation related behaviours in normative, strategic and operational managerial perspective. These characteristics influence the innovation capability in their unity, whereby both physical and intangible factors are playing important roles (Kramer et al., 2011). Hereby, the system-oriented approach of managing innovation capability provides a more holistic and fundamental overview on innovation as an element of the corporate innovation system.

The application of methods plays a significant role in executing of innovation related activities and consequently influences innovation capability, as several empirical studies suggest (Graner and Mißler-Behr, 2014; Franke et al., 2009). Following the systemic perspective, methods are a supporting factor to foster innovation capability by providing structures, behaviours and activities within the corporate innovation system as shown in Figure 1. 


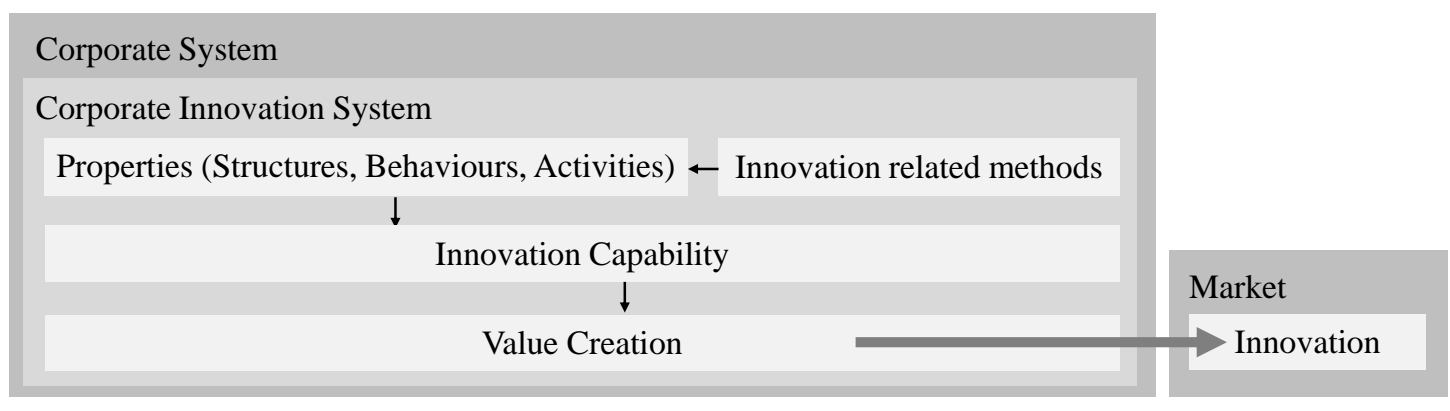

Figure 1. Alignment of methods within the corporate innovation system

Hereby, methods provide an operatively applicable thinking and behaviour pattern to achieve a goal (Vietor and Lachmayer, 2016). Thus, organizations would benefit by implementing methods as they provide standardized procedures for activities and thus give guidance and stability by reducing complexity and risks. Furthermore, methods support simplifying complex problems into solvable subproblems. Also, methods can serve as enablers of collaboration, as they standardize communication, decision making and documentation. In a general sense, methods have the potential to promote drivers and exploit potentials to foster innovation capability. However, applying methods involves critical aspects and challenges to be considered. Some methods are simple to apply, others require effort and experience to achieve the desired effects. The effort involved in method introduction and application and the fact that positive results are often not immediately visible, can lead to a scepticism about methods in practice. Thus, methods must be applied appropriately, purposeful, while considering the specific circumstances of respective situations and the individual appliers. (Lindemann, 2009). If that is the case, methods promise a number of positive effects to foster innovation capability.

\subsection{Current practice of method application to foster innovation capability of SMEs}

The various foci of differing understandings for innovation result in different approaches, methods and tools intending to foster innovation capability (Hidalgo and Albors, 2008). In addition, a diffuse understanding about fields to foster innovation capability exists especially in SMEs, which impacts the effectiveness of method application harmfully. In many cases, subjectively perceived potentials and challenges for innovation lead to misdirected target definitions to foster innovation capability and furthermore, they are coped with methods, that are being selected, based on limited method knowledge (Bavendiek et al., 2014) and problem understanding. Moreover, during application and implementation of methods organizational circumstances are considered insufficiently (Wallace, 2011). As a result, an efficient application of appropriate methods to foster innovation capability is often not realised in SMEs. Various sources, e.g. literature or digital portals, aim to provide innovation related methods. These approaches for method provision allocate methods according to predefined innovation activities or processes, which in particular do not exist in SMEs or are highly generic and thus are not implementable in the specific contexts of the appliers. Concluding, an effective clarification of the diffused understanding about innovation capability in SMEs and support for the application of methods are rarely given in practice and literature. Hence, the current practice addresses an essential need for consistent concepts to build up innovation awareness in SMEs and facilitate the application of methods to handle risk and exploit potentials.

\subsection{Research focus of this contribution}

Aim of this paper is to introduce a methodology to identify fields and methods for fostering innovation capability in SMEs as a preliminary step and support for successful method application for SME. The introduced methodology facilitates to build up an understanding of innovation in a company-specific context. Consequently, the enhanced understanding supports the definition of appropriate targets to cope with respective methods by considering the specific characteristics of SMEs. For this purpose this paper deals with following questions:

- How can SMEs be supported in building a specific innovation understanding as a basis for the application of methods to foster their innovation capability?

- Which fields and factors provide and support the innovation capability of SMEs? 
Therefore, primarily the main steps for the successful application of methods to foster innovation capability of SMEs are introduced within a holistic framework including recommendations for operationalization. This paper focuses on the development of a methodology for the initial step of the framework facilitating an awareness about company-specific fields to foster innovation capability. For the development of the methodology a model, named "InnoCheck Model", is introduced describing and structuring the innovation capability of SMEs. Based on the structure, fields and factors describing the innovation capability of SMEs are introduced. Concluding, case-studies applying the method in different manufacturing SMEs and the respective findings are discussed as a basis for further research and development of the proposed framework.

\section{A FRAMEWORK TO FOSTER INNOVATION CAPABILITY IN SMES}

The need for consistent concepts to support the method application in order to foster innovation capabilites of SMEs led to an initial literature research about current needs, challenges and potentials in order to answer the initial research question 'How can SMEs be supported in building up a specific innovation understanding as a basis for the application of methods to foster their innovation capability?'. For this purpose, an empirical analysis based on by semistructured interviews was carried out with various experts from different manufacturing SMEs companies to identify basic fields, challenges and needs to foster innovation capability (Şahin et al., 2018). The initial analysis with practitioners confirmed the facts found in literature regarding the diffuse understanding about the term and fields of innovation. The interviews also indicate the limited knowledge with regard to innovation related methods. In addition, innovation activities are often seen as an additional work load to daily business, so that innovation related methods are not properly implemented and applied in SMEs.

Due to the inconsistent application of innovation methods, basic steps for successful method application in general have been identified according to Lindemann (2009). This steps can be divided in selection, adaption and application of methods. An essential premise for the selection of appropriate methods is the clarification and definition of a specific issue to be solved or target to be reached. After defining a specific target, it is important to clarify whether the issue can be supported and which of the expected results can be reached using a particular method. After selecting an appropriate method, an implementation in the application context has to be realised. The method also needs to be adapted to the conditions during the application. The described steps were applied to the field of innovation capability in order to develop a framework for successful method application. Thus, regarding to the current situation of SMEs in order to foster innovation capability following steps can be defined:

- $\quad$ identification of fields and definition of issues to foster innovation,

- selection of methods which promise the expected output for predefined issues from given inputs,

- implementation of selected methods in the context of method appliers,

- consideration of circumstances during the method application.

The main steps are form a framework with corresponding aims of methodologies and tools to be developed, as shown in Figure 2.

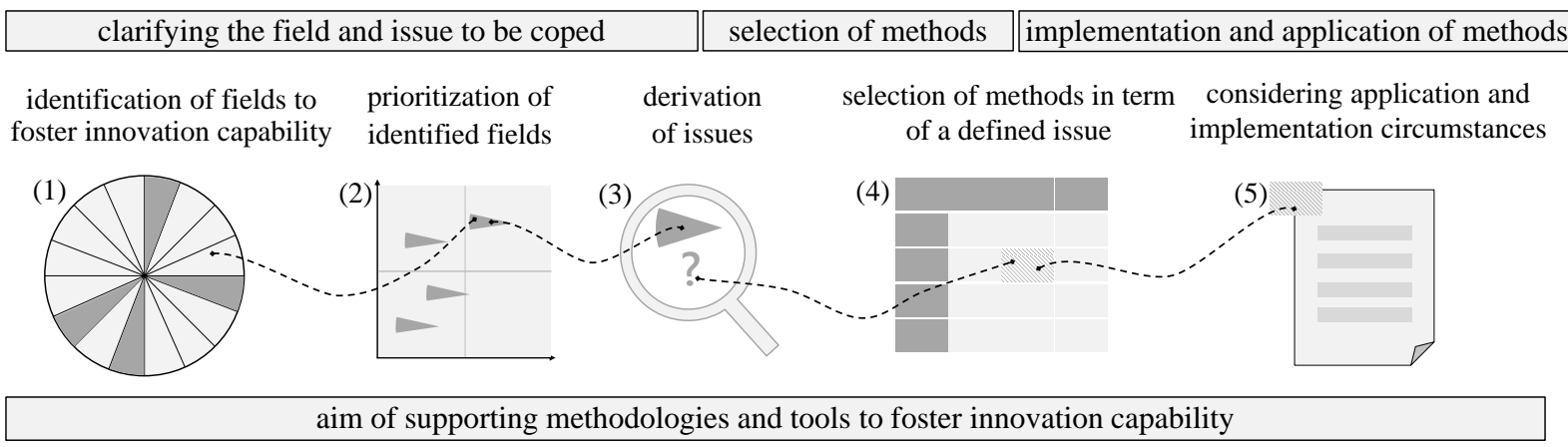

Figure 2. Framework with main steps for successful application of methods to foster innovation capability of SMEs 
Due to the diffuse understanding of innovation capability in SMEs, an identification of fields to foster innovation capability in the respective current state of the considered company hast to be executed as a first step of the framework for successful application of methods. This can be carried out by internal assessments and discussions about the current state and fields shaping the innovation capability of SMEs. This kind of assessment supports to build a specific understanding of innovation, existing strengths and weaknesses and relating activities to focus on. In order to focus on specific fields a prioritization of the identified fields needs to be carried out regarding their potentials to foster innovation activities of the specific SME in short and long-term perspective. The identified and prioritized fields facilitate the derivation and definition of specific issues to be coped for fostering innovation capability, which are primary steps to clarify the overall value of a method application and are the essential steps to select appropriate methods. The defined issues can then be coped within the step selection of provided methods. The subsequent method selection can be supported by a collection of methods impacting respective fields of innovation capability and providing selection criteria regarding specific implementation and application aspects. The methods can be provided in form of descriptions considering the implementation and application aspects of SMEs. As a result, the described steps and possible elements of the framework build a consistent approach for successful application of methods and outline possible areas for future research. The following section describes the development of a method supporting the first required step 'identifying fields to foster innovation capability of SMEs' particular method.

\section{INNOCHECK - A MODEL TO IDENTIFY FIELDS TO FOSTER INNOVATION CAPABILITY OF SMES}

The required method to identify the fields for fostering innovation of SMEs led to the question 'Which fields and factors provide and support innovation capability of SMEs' and a subordinate research question 'How can SMEs be supported in identifying fields to foster their innovation capability?'. To support identifying innovation fields, a certain knowledge structure was developed as a fundamental framework in form of a potential model proposed earlier in Şahin et al. (2018) and Inkermann et al. (2017). The proposed structure enables to classify different knowledge types regarding innovation capability in SMEs and facilitates a semiformal process to identify fields to foster innovation capability. The structure, knowledge levels and semiformal process is shown in Figure 3.

Strategic level (1)

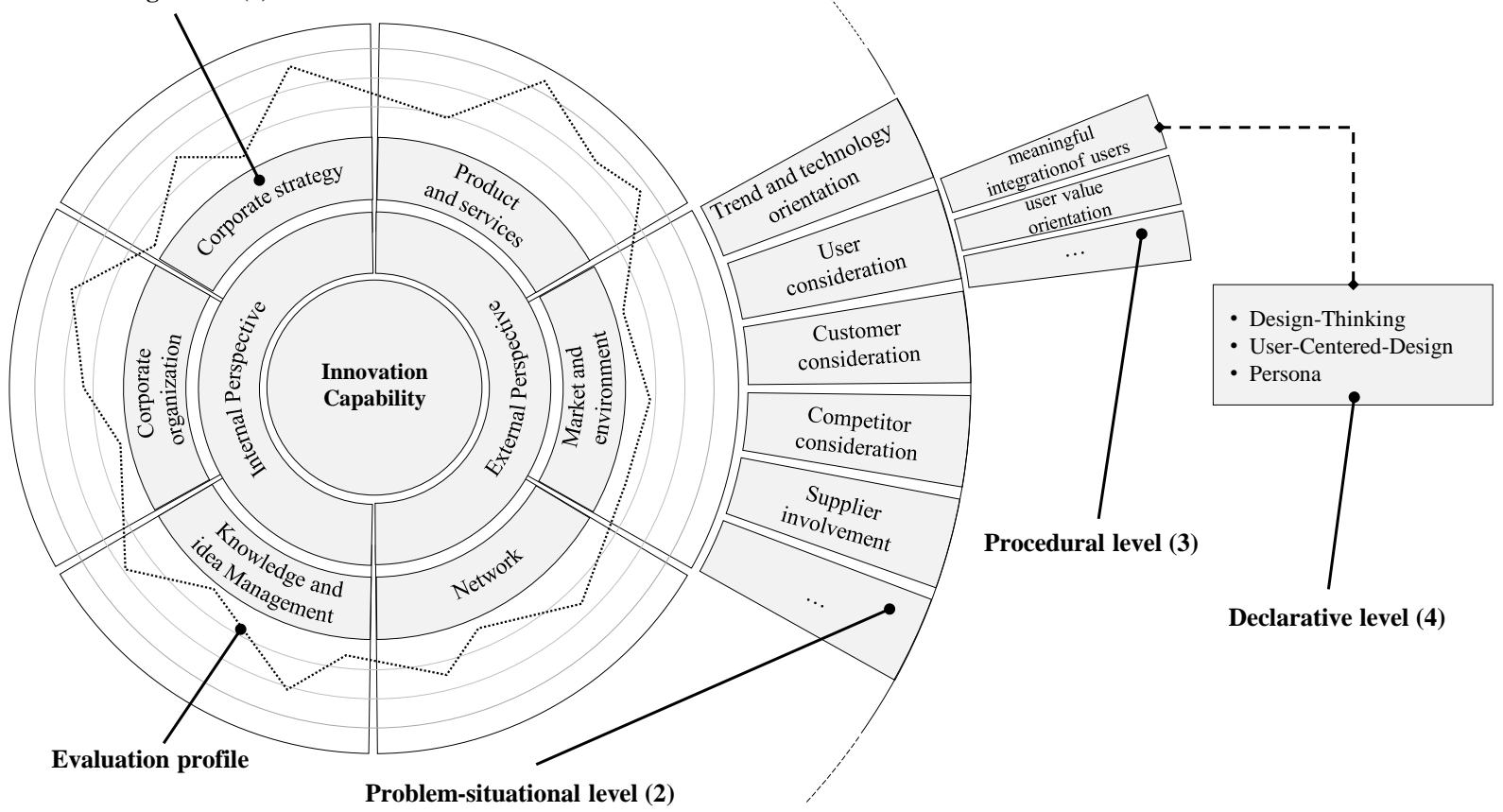

semiformal methodology

Figure 3. Methodology to identify fields for innovation and collaboration for SMEs 
The introduced model indicates different field to foster innovation capability in SMEs by structuring four types of knowledge according to Jong and Ferguson-Hessler (1996), which have been applied to field of innovation capability in Şahin et al. (2018). The levels are:

1. Strategic knowledge: Least domain specific level of innovation capability, describing strategic fields of SMEs innovation capabilities.

2. Problem-situational knowledge: Problem oriented fields within the strategic levels which indicate relevant fields of action and support the assessment of SMEs innovation capability.

3. Procedural knowledge: Procedural indicators that are specific enough for the derivation of specific measures and assessment of problem-situational fields.

4. Declarative knowledge: Detailed instructions for specific measures within the problemsituational e.g. methods descriptions that can be applied as a result.

The introduced structure and model provides a semiformal method to identify fields and methods to foster innovation in SMEs. The assessment can be realised by rating the activities of the considered company on the procedural-level (3). Based on the rating a profile is emerging indicating the innovation capability also from a strategical (1) and procedural (2) point of view. Based on the evaluation profile and insights during the assessment first fields to initiate fostering the innovation capability can be identified. This profile allows a simplified overview of the innovation capability on different concretization levels, allowing the discussion of possible fields and the derivation of specific issues based on the current situation of the company. Within the declarative level (4) methods can be assigned to the respective fields. Further explanations about the model structure and the details of the semiformal method can be found in Şahin et al. (2018).

The second research question 'Which fields and factors provide and support innovation capability of $S M E s$ ' was addressed to elaborate the specific content of the model in terms of innovation capability. To establish an elementary guidance for the contentual development, an initial literature research was conducted regarding strategic fields to foster the innovation capabilities of SMEs. During the initial literature review existing audits for SMEs were analysed, which served as orientation for first expert interviews in SMEs. The interviews have been executed with mainly general or innovation managers of manufacturing SMEs in Lower Saxony, Germany. Main topics of the interviews were the identification of needs, challenges and experiences with regard to the field of innovation and innovation capability of SMEs. Based on the literature research and the interviews initial fields within the model could be identified, which served also as a guidance for a systematic literature review. The systematic literature review was carried out to identify and describe fields and factors that influence the innovation capability in SMEs and their appropriate mapping within the levels of the potential model. In total a number of 17 sources were identified that holistically consider and describe innovation capability of SMEs at various levels, for instance Keizer et al. (2002), Pierre and Fernandez (2018). Sources which are focusing on specific perspectives, for instance radical innovation capability (O'Connor et al., 2008), or just describing influencing aspects for one specific field, for instance networks (Mohannak, 2007), have not been included. These need to be taken into consideration later in the detailed elaboration and evaluation of the individual fields. After selecting the respective sources, the fields and factors have been assigned to corresponding levels respectively to the knowledge types of the model. As a result, a comprehensive version of fields and factors to foster innovation capability were identified and assigned to the model. Based on this, the developed model was supplemented with the data from the initial interviews. The resulting potential model was optimized according to requirements (Herstatt et al., 2007) for tools to assess innovation capability of SMEs. The derived requirements in terms of the methodology are:

- Holism: holistic approach to describe or measure innovation capability

- Comprehensibility and Validity: The field and factors should be logically structured as well as validated with empirical data,

- Suitability for SMEs: non-applicable or not-understandable dimension for SMEs should be removed or adjusted, as SMEs possess characteristic that may differ from larger organisations,

- Contextual Suitablity: As the industry and context varies, the methodology shall fit to the scope and SMEs context, in this case manufacturing industry.

The resulting content of the model is shown in Figure 4, addressing the initially research question 'Which fields and factors provide and support innovation capability of SMEs. 


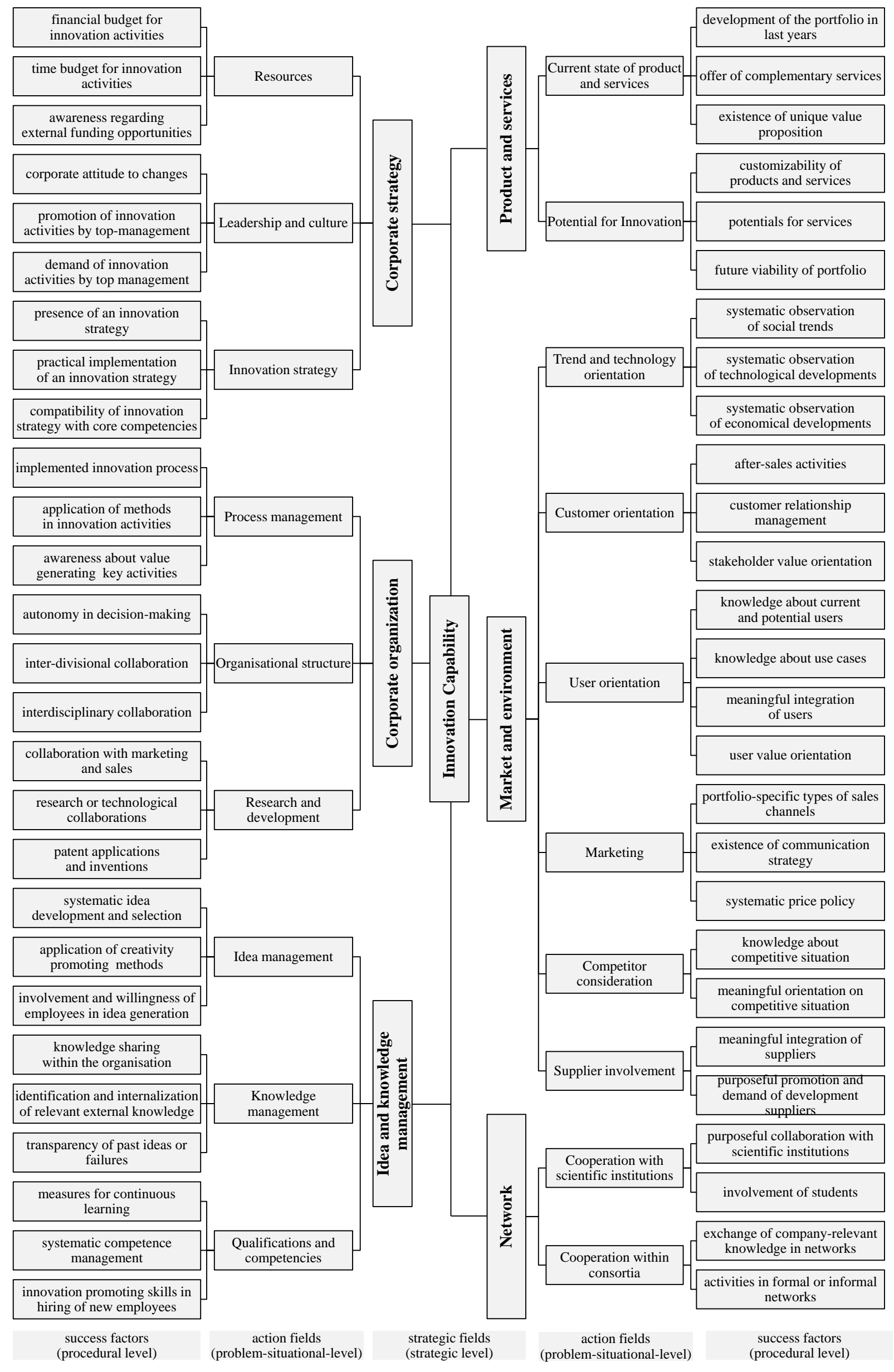

Figure 4. Field and factors providing the innovation capability of SMEs 


\section{APPLICATION AND EVALUATION OF THE METHOD TO IDENTIFY FIELDS TO FOSTER INNOVATION CAPABILITY}

In this section, the application and evaluation of the introduced method are discussed. The application of the method was carried out in moderated workshops with seven SMEs from the bussiness-to-bussines and bussiness-to-customer sector in the automotive, steel and rail vehicle industries as well as electronic and safety systems segments. Overall aim of participating companies was to identify of companyspecific fields to foster innovation capability as a first step before defining issues and selecting appropriate methods. From a scientific point of view, the execution of the methodology and respective transfer as well as resulting data and the impact on the participants were evaluated by surveys and qualitatively in form of action research. Participants came from first and second management levels and from different areas, thus ensuring a broad coverage of levels of responsibility and professional competences as well as an in-depth knowledge of their corporate history, culture and strategy. As a primarily sensitization regarding the innovation capability, the strategical and action fields as well as success factors influencing the innovation capability were introduced to the participants. In order to reflect the current innovation capability, the respective participants were asked to assess the different action fields within the potential model with regard to their individual estimation. The introduced success factors within the model served as references for the estimation of the actions fields. The evaluation was separated into an initially individual assessment and following common discussion. During the common discussion, the averages of the individual estimations for the respective fields were compiled and the reasons for different estimations, challenges and needs in respective fields were discussed. Subsequently, the desired state in a long-term perspective for each action field was asked. The resulting gaps between the current and long-term desired state in the respective fields were identified as possible field for further analysis in order to define issues to foster innovation capability and ultimately to cope with methods. At the end of the workshops, evaluation sheets were handed over to all workshop participants for evaluating aspects of execution of the methodology, respective transfer, resulting data and the impact on the participant. In total 29 evaluation sheets could be obtained. As an exemplary result Figure 5 shows the current and desired innovation capability of a participating company from the steel construction sector.

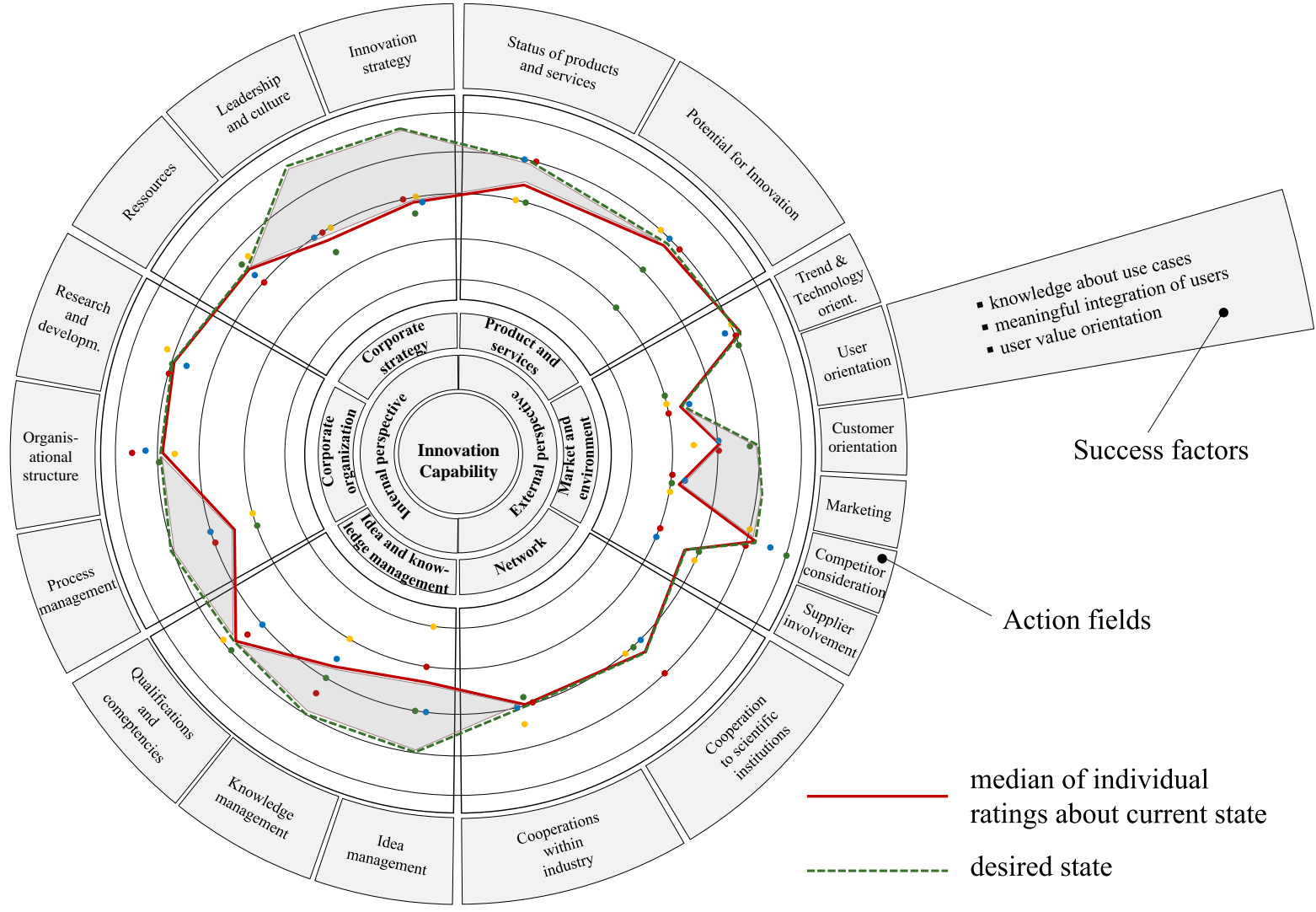

Figure 5. Results of a case study - innovation capability of a SME from the steel construction sector 
The application of the developed method led to an expressive result about the current state and companyspecific fields to foster innovation capability. As an outcome the action fields of innovation strategy and leadership, idea and knowledge management as well as customer orientation and marketing could be identified as particularly relevant for the sample SMEs by considering the gaps between the current and the desired states. A prioritization of the identified field is required in the next step for the definition of the issue to foster innovation capability and the selection of appropriate methods.

Using the introduced methodology for the identification of fields to foster innovation capability has been conveyed as advisable. The participants expressed that innovation is usually tied to specific aspects such as products or processes, rather than a holistic understanding. By applying the methodology, a new understanding of fostering innovation capability in a holistic way throughout the corporate innovation system was established. Applying the model also arose energetic discussions among the appliers which provided a common understanding about specific challenges and potentials. Furthermore, these discussions were crucial to motivate them for involving in future steps. Thus the individual assessment has been received positively since it created discussions and also contributed to the overall result. Consequently, the introduced methodology can also be a valuable element for influencing a company's innovation culture. One key success contributor to the model is that participants of the companies are in control over the process of assessing and identifying fields for innovation in comparison to typical innovation audits by external professionals. As a result, fields to foster innovation could be identified in a quantitative way by the assessment and in a qualitative way during the accompanying discussions.

Results from the evaluation surveys and feedbacks point out that the application of the method was easily understood. Based on the evaluation results, some implications for further improvements could be derived. Some of the action fields and success factors were not equally understood by all participants, thus a respective explanation was expressed to be helpful. The need for more practical examples in form of best practices to explain and motivate the respective fields and factors was also expressed. The totality of SMEs' innovation capability profiles confirm that the methodology leads to individual results. The results so far indicate that the potentials of external knowledge, especially of users and external organizations, are not being exploited effectively and are mostly not considered as relevant.

It must be emphasized, that the methodology has been developed with a focus on SMEs that are developing, producing and selling technical consumer or industrial products mainly from the mechanical domain. Thus, our model is contextually limited as empirical studies to develop the methodology are constrained in this manner. Furthermore, the results and implications are dependent on the characteristics of their applicants as well as the specific application settings.

Summarized, we conclude that the proposed methodology supports SMEs to identify fields to foster innovation capability. Additionally, the methodology can be exploited as a methodology for changing the innovation mind-set and culture within SMEs.

\section{DISCUSSION AND CONCLUSION}

A significant driver for innovation in SMEs is a systemic view on innovation capability and its fostering by methods. In this contribution, a framework proposing main steps for successful method application to foster innovation capability of SMEs is presented. As a first step of the framework a methodology and a model to identify fields to foster innovation capability of SMEs is introcued. Furthermore, fields and factors on different levels influencing the innovation capability of SMEs are presented. Our findings show that the application of the introduced methodology leads to an enhanced awareness, holistic understanding and valuable discussions about company-specific fields to foster innovation capability in SMEs. Concluding, case studies discussing the application of the introduced method are presented.

The proposed framework still requires subsequent steps for the successful application of methods to foster innovation capability. This is because the identified field might be not specific enough, since more than one field can seem relevant to apply methods. Therefore, it necessitates a methodology for prioritizing identified fields and a subsequent definition of issues to be coped by methods to foster innovation capability as proposed in the framework. Thus, further work will focus on the development of the proposed framework which aims to facilitate a successful method application in SME to bring out innovation. The applications settings of the methodology are based on limited amount of experienced workshops. It hence requires further research and a wider range of workshops to identify related factors influencing its application in order to improve the respecitve application guidelines. 


\section{REFERENCES}

Baregheh, A., Rowley, J. and Sambrook, S. (2009), "Towards a multidisciplinary definition of innovation”, Management Decision, Vol. 47 No. 8, pp. 1323-1339, http://doi.org/ 10.1108/00251740910984578.

Bavendiek, A.-K., Inkermann, D. and Vietor, T. (2014), "Konzept zur Methodenbeschreibung und -auswahl auf Basis von Kompetenzen und Zusammensetzung von Entwicklungsteams", in Krause, D., Paetzold, K. and Wartzack, S. (Eds.), Design for X: Beiträge zum 25. DfX-Symposium, Erlangen, pp. 215-226.

Bleicher, K. (1999), Das Konzept integriertes Management: Visionen - Missionen - Programme, St. Galler Management-Konzept, Bd. 1, 5., rev. und erw. Aufl., Campus-Verl., Frankfurt/Main, New York.

Cooper, J.R. (1998), “A multidimensional approach to the adoption of innovation", Management Decision, Vol. 36 No. 8, pp. 493-502, http://doi.org/10.1108/00251749810232565.

Cooper, R.G. (2001), Winning at new products: Accelerating the process from idea to launch, 3rd ed., Perseus Pub, Reading, Mass.

Eversheim, W. (2009), Innovation management for technical products: Systematic and integrated product development and production planning, RWTHedition, Springer, Berlin, Heidelberg, http://doi.org/10.1007/978-3-540-85727-3.

Franke, S., Kirschner, R., Kain, A., Becker, I. and Lindemann, U. (2009), "Managing early phases of innovation processes and the use of methods within - Empirical results from an industry survey", in DS 58-1: Proceedings of ICED 09, the 17th International Conference on Engineering Design, Vol. 1, pp. $193-204$.

Graner, M. and Mißler-Behr, M. (2014), "Method application in new product development and the impact on cross-functional collaboration and new product success", International Journal of Innovation Management, Vol. 18 No. 01, p. 1450002, http://doi.org/10.1142/S1363919614500029.

Herstatt, C., Buse, S., Trapp, S. and Stockstrom, C. (2007), Leistungsmerkmale eines KMU-gerechten Innovationsaudits - Beitrag zur Erarbeitung eines Hamburger Innovationsaudits, TUHH Universitätsbibliothek, http://doi.org/10.15480/882.279.

Hidalgo, A. and Albors, J. (2008), "Innovation management techniques and tools: a review from theory and practice", R\&D Management, Vol. 38 No. 2, pp. 113-127, http://doi.org/10.1111/j.1467-9310.2008.00503.x.

Inkermann, D., Kleemann, S. and Vietor, T. (2017), "Ein Potentialmodell für die Nutzung neuer Technologien in der Produktentwicklung”, in Binz (Eds.), Stuttgarter Symposium für Produktentwicklung, Stuttgart.

de Jong, T. and Ferguson-Hessler, M.G.M. (1996), "Types and qualities of knowledge”, Educational Psychologist, Vol. 31 No. 2, pp. 105-113, http://doi.org/10.1207/s15326985ep3102_2.

Keizer, J.A., Dijkstra, L. and Halman, J.I.M. (2002), "Explaining innovative efforts of SMEs”, Technovation, Vol. 22 No. 1, pp. 1-13, http://doi.org/10.1016/S0166-4972(00)00091-2.

Kramer, J.-P., Marinelli, E., Iammarino, S. and Revilla Diez, J. (2011), "Intangible assets as drivers of innovation. Empirical evidence on multinational enterprises in German and UK regional systems of innovations", Technovation the international journal of technological innovation, entrepreneurship and technology management, Vol. 31 No. 9, pp. 447-458.

Lindemann, U. (2009), "Methodische Entwicklung technischer Produkte: Methoden flexibel und situationsgerecht anwenden", VDI-Buch, 3., korrigierte Aufl., Springer, Berlin.

Mohannak, K. (2007), "Innovation networks and capability building in the Australian high-technology SMEs", European Journal of Innovation Management, Vol. 10 No. 2, pp. 236-251, http://doi.org/10.1108/14601060710745279.

O’Connor, G.C., Paulson, A.S. and DeMartino, R. (2008), “Organisational approaches to building a radical innovation dynamic capability", International journal of technology management, Vol. 44 No. 1/2

Pierre, A. and Fernandez, A.-S. (2018), “Going Deeper into SMEs' Innovation Capacity: An Empirical Exploration of Innovation Capacity Factors", Journal of Innovation Economics, Vol. 25 No. 1, p. 139, http://doi.org/10.3917/jie.pr1.0019.

Rosenbusch, N., Brinckmann, J. and Bausch, A. (2011), "Is innovation always beneficial? A meta-analysis of the relationship between innovation and performance in SMEs", Journal of Business Venturing, Vol. 26 No. 4, pp. 441-457, http://doi.org/10.1016/j.jbusvent.2009.12.002.

Şahin, T., Cudok, A., Rapp, S., Inkermann, D., Albers, A., Wattenberg, F., Bursac, N. and Vietor, T. (2018), "How to foster innovation? Findings and hypotheses for collaborations between research and industry", in Proceedings of International Design Conference, DESIGN, Vol. 4, pp. 1945-1956, http://doi.org/10.21278/idc.2018.0484.

Schumpeter, J.A. (1934), "The theory of economic development: An inquiry into profits, capital, credit, interest, and the business cycle", Harvard economic studies, Vol. 46, 2nd print. d. ed. 1934, Harvard Univ. Press, Cambridge, Mass.

Vietor, T. and Lachmayer, R. (2016), Massive Open Online Course "Methods and Tools for Engineering Design", Lecture notes and videos.

Wallace, K. (2011), “Transferring Design Methods into Practice”, in Birkhofer, H. (Ed.), The Future of Design Methodology, Springer London, London, pp. 239-248, http://doi.org/10.1007/978-0-85729-615-3_21. 